\title{
L'affaire de la mémoire de l'eau. Pour une sociologie de la communication scientifique
}

In: Réseaux, 1993, volume 11 n58. pp. 67-89.

\begin{abstract}
Résumé
Cet article met en évidence les médiations complexes qui relient le champ scientifique et ses produits légitimes - les articles des revues spécialisées - aux autres circuits de l'information scientifique, à l'espace public mass-médiatique en particulier. La controverse à propos de "l'affaire de la mémoire de l'eau » donne l'occasion d'étudier les réseaux socio-techniques qui soutiennent la construction des faits scientifiques et en permettent la circulation au-delà de l'enceinte des laboratoires. Elle incite à dépasser une approche de type internaliste ou externaliste de l'activité scientifique. Elle fournit des éléments de compréhension des mutations qui affectent le système de communication et de coordination du champ scientifique contemporain.
\end{abstract}

\section{Abstract}

This article highlights the complex mediation linking the scientific field and its legitimate products - articles in specialized reviews to other networks of scientific information, and to the public area of mass-media in particular. The controversy around the "memory of water affair" provides an opportunity to study the socio-technical networks on which scientific facts are based and which permit their circulation beyond the confines of laboratories. It prompts one to go beyond an internalist or externalist type of approach to scientific activity, and provides elements for understanding the mutations which affect the communication and coordination system of the contemporary scientific field.

Citer ce document / Cite this document :

Kaufmann Alain. L'affaire de la mémoire de l'eau. Pour une sociologie de la communication scientifique. In: Réseaux, 1993, volume $11 \mathrm{n}^{\circ} 58$. pp. 67-89.

http://www.persee.fr/web/revues/home/prescript/article/reso_0751-7971_1993_num_11_58_2305 


\section{L'AFFAIRE DE LA MÉMOIRE DE L'EAU}

pour une sociologie de la communication scientifique

Alain KAUFMANN 
$\mathrm{L}$ a sociologie des sciences, y compris dans ses développements les plus récents, a fort peu abordé le problème du rôle joué par la « publicité » faite aux découvertes scientifiques sur la scène médiatique dans le cadre de controverses portant sur des résultats de recherche contestés et/ou hérétiques. Nous voulons tenter d'analyser ici les médiations complexes qui relient le champ scientifique et ses produits légitimes - les articles des revues spécialisées - aux autres circuits d'information scientifique, à l'espace public mass-médiatique en particulier. L'analyse des controverses à propos de «l'affaire de la mémoire de l'eau » (1), au travers de l'examen parallèle de la littérature spécialisée et de la grande presse, fournit une excellente occasion de mettre en évidence ces mécanismes de contamination qui nous incitent à dépasser les approches unilatérales de type internaliste ou externaliste de la science. Nous aurons également l'occasion de mentionner quelques autres « af- faires » récentes qui jettent une lumière intéressante sur le problème de l'information scientifique.

La controverse sur les effets des hautes dilutions, sur laquelle la sanction de l'Histoire ne s'est pas encore abattue, nous permet de satisfaire à un critère méthodologique dont de nombreux travaux récents ont montré la pertinence : étudier les controverses non encore closes ou encore «la science en train de se faire » (2) et... de se défaire.

Nous commencerons par exposer les principaux éléments et enjeux de l'affaire. Nous retracerons ensuite brièvement la naissance du système de communication des faits scientifiques, ce qui nous permettra de nous interroger sur le rôle joué par les revues spécialisées. Nous examinerons ensuite comment la littérature "profane » vient s'articuler sur cette littérature «sacrée » dans le processus de construction des faits scientifiques. Nous tenterons enfin de montrer que l'on ne gagne rien à vouloir interpréter les multiples recouvrements entre les différents forums de l'information scientifique en termes de pathologie ou de dysfonctionnement. Les traductions foisonnantes et contradictoires qui sont faites de la mémoire de l'eau par l'intermédiaire des divers canaux d'information nous donnent l'occasion de reconstruire les réseaux socio-techniques sans lesquels les faits scientifiques ne peuvent se propager au-delà de l'enceinte des laboratoires.

\section{L'AFFAIRE DE LA MÉMOIRE DE L'EAU}

Dans le numéro daté du 30 juin 1988 de la très prestigieuse revue britannique $\mathrm{Na}$ ture paraît un article intitulé : "Dégranulation des basophiles humains induite par de très hautes dilutions d'un antisérum

(1) Cette affaire a fait l'objet, de la part de l'auteur de cet article, d'un travail de recherche non publié, fondé sur l'examen exhaustif des articles parus dans Nature, Science, Current Contents, ainsi que dans Le Monde, Libération et Science et Vie. Ce document contient une bibliographie complète de ces sources. Des exemplaires de ce travail sont disponibles auprès de l'auteur. On trouvera également chez DE PRACONTAL, 1990, ainsi que chez ALFONSI, 1989, de nombreux renseignements complémentaires, en particulier des interviews des principaux protagonistes. Le numéro 13 des Current Contents daté du 27 mars 1989 contient également un inventaire de la littérature portant sur cette controverse dans la littérature anglo-saxonne.

(2) Pour un exposé détaillé des implications de ce précepte, cf. LATOUR, 1989 a. 
anti-IgE » (3). Cet article de trois pages est cosigné par treize auteurs appartenant à cinq laboratoires. Deux sont situés en Israël, un en Italie, un au Canada et un en France. Le laboratoire français, qui abrite l'unité 200 de l'Institut national de la santé et de la recherche médicale (INSERM), implanté à Clamart dans la région parisienne, est le maître d'œuvre et le coordonateur de l'étude. Il est spécialisé dans la recherche sur les phénomènes allergiques et inflammatoires. Son directeur, le docteur Jacques Benveniste, va se retrouver au centre d'une vaste polémique qui va enflammer le monde de la recherche et la presse internationale.

\section{D'étranges expériences}

Afin de bien percevoir les enjeux qui sont sous-tendus par ces travaux, il est indispensable d'exposer avec quelque détail en quoi consiste cette série d'expériences et les phénomènes biologiques qui s'y rapportent. Comme l'ont bien montré les développements récents de la sociologie des sciences et des techniques, ce n'est qu'en développant une compréhension interne des pratiques scientifiques, y compris dans leurs composantes les plus « dures », qu'on peut en saisir à la fois les spécificités et les parentés avec d'autres champs de la culture.

Les patients allergiques présentent dans leur sang une production anormalement élevée d'un type particulier d'anticorps (4) appelé immunoglobuline $\mathrm{E}$ (IgE). Ces anticorps sont dirigés de manière spécifique contre une série de substances naturelles, ou allergènes, qui constituent les facteurs déclenchant des phénomènes allergiques. Ces allergènes sont classiquement représentés par les poussières, les pollens, les acariens, etc.

Les IgE se trouvent attachées à la surface de certains globules blancs du sang nommés basophiles, où elles jouent le rôle de récepteurs. Lorsqu'un allergène entre en contact avec ces récepteurs, il provoque la libération par les basophiles d'une série de substances contenues à l'intérieur de petites vésicules ou granules. On parle alors de dégranulation des basophiles. L'une de ces substances, l'histamine, se retrouve ainsi dans le courant sanguin et provoque une série de symptômes caractéristiques des phénomènes allergiques.

Il est possible d'évaluer in vitro le statut allergique d'un patient au moyen d'un test standard. On prélève pour cela un échantillon de sang auquel on ajoute un colorant : le bleu de toluidine. Cette substance a pour effet de colorer en bleu les basophiles et en rouge les granules qu'ils contiennent. On ajoute ensuite l'allergène que l'on désire tester et l'on observe le résultat de l'opération au microscope. Si les granules rouges sont toujours visibles à l'intérieur des cellules, on en déduit que l'allergène en question n'a pas provoqué de réaction allergique. Par contre, si les cellules apparaissent totalement bleues, c'est que les basophiles ont subi une dégranulation, libérant ainsi leur contenu en histamine dans le sang, que l'on peut ensuite doser. Pour chaque échantillon observé, il est ainsi possible de quantifier l'intensité de la réaction allergique en calculant un pourcentage exprimant le rapport entre le nombre de cellules qui ont dégranulé et celles qui ne l'ont pas fait. Dans les expériences controversées, on a remplacé l'allergène par un antigène anti-IgE humaine tiré du sang de chèvre.

L'ensemble des procédures que nous avons décrites jusqu'ici représente des expériences tout à fait classiques. La particularité du protocole introduit par l'équipe de l'INSERM réside dans le fait d'utiliser non pas des solutions concentrées d'anti-IgE de chèvre, mais des solutions hautement diluées. Le processus a été poussé jusqu’à diluer 60 et même 120 fois la solution de départ, ce qui pose un problème crucial. En effet, pour la physique et la chimie, une solution d'une concentration donnée

(3) DAVENAS et al., 1988.

(4) Les anticorps sont des protéines participant au processus de défense immunitaire. Leur production est induite par l'introduction dans l'organisme d'une substance reconnue comme étrangère, ou antigène. Les allergènes constituent une classe particulière d'antigènes. 
contient un nombre fini de molécules, exprimé par une grandeur appelée nombre d'Avogadro. Dès la quinzième dilution, on ne fait donc en principe que diluer de l'eau avec de l'eau. Pour donner une image plus parlante de la situation, disons que, si l'on est parti d'une solution initiale contenant 1 gramme d'IgE par litre de solution, à ce stade déjà, on obtient en moyenne un gramme d'IgE pour 100 milliards de mètres cube d'eau.

Ce qui est tout à fait surprenant, c'est que, après 120 dilutions successives, l'équipe de l'INSERM observe toujours une dégranulation des basophiles humains, alors que la solution ne contient théoriquement plus aucune molécule d'anti-IgE de chèvre depuis longtemps. Il semble donc que l'on observe un «effet moléculaire sans molécules ». Comme si la solution d'eau avait conservé l'empreinte ou la mémoire des molécules. Autre observation troublante, le pourcentage de dégranulation observé lorsque l'on augmente les dilutions ne diminue pas, mais montre une série d'oscillations qui semblent relativement indépendantes de la dilution. De plus, l'effet des hautes dilutions n'est observé que si la solution est vigoureusement agitée.

\section{L'ombre de l'homéopathie}

Pour évaluer la portée potentielle de ces observations, qui semblent contredire les principes élémentaires de la causalité physico-chimique, il nous faut dès à présent souligner la parenté qu'elles entretiennent avec le domaine de l'homéopathie. Cette pratique, qui est rattachée à la vaste constellation des «médecines douces » ou « médecines naturelles», projettera en effet continuellement son ombre sur la controverse.

Le fondateur de l'homéopathie est le médecin allemand Samuel Hahnemann, né à Meissen en 1755 et mort à Paris en 1843. A la suite d'expérimentations pratiquées sur sa propre personne, il énonça le principe fondateur de la pratique homéopathique : le «principe ou loi de similitude ». Sur la base de ce principe, déjà présent dans le corpus hippocratique, il est pos- sible de définir l'homéopathie comme une méthode thérapeutique qui utilise à dose non toxique, chez un individu malade, des substances médicamenteuses capables de déclencher à dose toxique ou subtoxique, chez un individu sain, un ensemble de symptômes semblables à celui de la maladie observée.

Par dose non toxique il faut entendre concentrations faibles ou très faibles de substance active, se situant souvent audelà de la limite fatidique du nombre d'Avogadro. En outre, pour être efficace, la préparation demande une vigoureuse agitation, que les homéopathes nomment «dynamisation ». On perçoit aisément, dès lors, les parallèles que l'on peut tracer, et qui seront souvent évoqués par la suite, entre l'expérience portant sur les effets des hautes dilutions d'IgE et la pratique homéopathique. Soulignons que, nulle part dans l'article de Nature, l'homéopathie n'est mentionnée.

Nous allons maintenant retracer la succession des principaux événements qui ont jalonné le déroulement de l'affaire.

\section{L'entrée en scène de Jacques Benveniste}

Nous remonterons jusqu'en mars 1985 , pour voir apparaître sur la scène médiatique le personnage qui bientôt se retrouvera au centre de cette affaire : le docteur Jacques Benveniste. Directeur de l'unité 200 de l'INSERM, il a autorisé depuis le début des années 80 que, dans le cadre de son laboratoire, se déroulent des expériences portant sur les effets cellulaires de substances hautement diluées couramment utilisées en homéopathie. Elles sont désignées, selon la tradition, par des noms latins comme Apis Mellifica, Belladona, Ferrum phosphoricum ou Silicea. Ces études sont menées dans le cadre d'un contrat entre les Laboratoires Homéopathiques de France, qui seront rachetés ultérieurement par les laboratoires Boiron, et l'INSERM. La participation de cet organisme national constitue sur le plan scientifique un gage de légitimité et de sérieux.

Les expériences ont été initiées par un jeune médecin homéopathe, Bernard Poi- 
tevin, bientôt rejoint par une biochimiste, Elisabeth Davenas, qui deviendra par la suite la spécialiste de la technique utilisant la dégranulation des basophiles. Ces chercheurs sont tous deux cosignataires de l'article de Nature.

Ces travaux semblent démontrer que les produits homéopathiques cités plus haut sont capables d'inhiber les réactions de certaines cellules, dont les basophiles, impliquées dans les processus allergiques, ce qui est conforme au principe de similitude. Les travaux publiés par Nature porteront quant à eux sur la stimulation de la réaction allergique et non pas sur son inhibition, ce qui les éloigne quelque peu du paradigme homéopathique. Cette opposition, inscrite à l'intérieur du protocole expérimental, permet à la fois aux auteurs d'accentuer certaines caractéristiques du phénomène et d'orienter l'interprétation de leurs lecteurs.

Dans l'article du Monde qui expose les résultats pour la première fois à un large public, Jacques Benveniste déclare : «Lorsque j'ai accepté de tester ces différents produits homéopathiques, j'étais très sceptique. Je ne connaissais rien à l'homéopathie, et ma culture scientifique - je dirais même scientiste - m'incitait plutôt à penser que l'homéopathie n'était qu'un placebo. D'où ma grande surprise à la vue des premiers résultats. [...] Il ne s'agit surtout pas d'en tirer des conclusions quant à l'efficacité thérapeutique de ces différents produits. [...] Que voulez-vous, c'est comme ça. On n'y peut rien. C'est un débat qui va sans doute me dépasser, qui me dépasse peut-être déjà. Mais les faits sont là (5). » On ne peut que souligner le caractère prémonitoire de la déclaration de Benveniste qui, plus de trois ans avant la publication finale dans Nature, entrevoit déjà le caractère de «bombe épistémologique » (6) que recèlent ces travaux.

Nous retrouvons Jacques Benveniste en décembre 1985 dans le cadre d'une conférence de presse donnée par le ministre des
Affaires sociales, Georgina Dufoix, et à laquelle participe également le directeur général de l'INSERM. Cette rencontre est organisée dans le but d'exposer les mesures gouvernementales visant à favoriser l'évaluation scientifique des médecines parallèles, dont l'homéopathie. La présence de Jacques Benveniste y est attestée de la manière suivante par l'un des chroniqueurs scientifiques de Libération, Eric Conan : «Et lorsque le docteur. Benveniste s'est permis de faire un hallucinant numéro de prestidigitation digne d'un cabaret borgne, montrant à la va-vite des graphiques illisibles (et refusant avec mépris tout commentaire) pour affirmer que les vertus de l'homéopathie étaient établies, un moment de panique saisit tout le monde. Le directeur général de l'INSERM tenta en catastrophe de rattraper le coup en réprimandant brutalement et immédiatement en public le brave Benveniste (7). »

\section{La longue marche vers la publication}

Sur la base des résultats obtenus, Jacques Benveniste décide, en juin 1986, soit deux ans avant la publication, de soumettre un article à la revue Nature, dont le rédacteur en chef, John Maddox, va jouer un rôle central dans la suite des événements. Il faudra environ dix-huit mois de négociations serrées et parfois tendues entre les différentes parties pour que l'article soit finalement accepté et publié. Il sera examiné au total par cinq référents (8). En résumé, les experts désignés ne trouveront rien à redire sur le fond, mais feront remarquer que les résultats présentés sont tout simplement « incroyables » et doivent s'expliquer par une quelconque erreur de manipulation, ce qui est aussi l'avis de Maddox.

Benveniste subit un premier refus, qui n'est pas motivé. Mais il ne se laisse pas décourager et réclame des explications. En novembre 1986, John Maddox soumet la

(5) Le Monde, mercredi 6 mars 1985. C'est nous qui soulignons.

(6) Nous empruntons cette expression à DE PRACONTAL, 1990, 21.

(7) Libération, mercredi 11 décembre 1985.

(8) Nous utiliserons ce mot en lieu et place du terme anglais de « referee ». 
publication à la condition préalable que les expériences soient reproduites de manière indépendante par d'autres laboratoires. Dans le courant de l'année 1987, deux laboratoires israéliens, situés à Rehovot, vont donc, à la demande de Benveniste, répéter les expériences. Ce travail s'effectuera avec la collaboration d'Elisabeth Davenas, détachée à l'étranger pour l'occasion. Sa présence sera à l'origine d'une atmosphère souvent pesante où le mot de fraude sera plusieurs fois prononcé. Une autre confirmation sera apportée dans le courant de la même année par une équipe milanaise et une équipe canadienne de Toronto, sous la forme de résultats préliminaires.

Au début de 1988, Benveniste adresse les résultats d'Israël et de Toronto à $\mathrm{Na}$ ture. L'article a été entre-temps totalement remanié en tenant compte des premières critiques formulées par les référents. En avril 1988, Maddox signifie à Benveniste qu'il accepte de publier pour autant que l'article soit accompagné d'un commentaire émanant des référents.

Le 27 mai 1988, dans le cadre du Congrès national d'homéopathie qui se déroule à Strasbourg, Benveniste fait une communication très remarquée concernant ses travaux sur les hautes dilutions. On y parle d'une « organisation de la matière actuellement inconnue ", de "molécules fantômes », « d'empreintes moléculaires », d'une eau qui aurait conservé le « souvenir » des molécules avec lesquelles elle aurait été en contact. Benveniste déclare : «J'observe, mais je ne comprends pas : nous ne pouvons fournir d'explication. Je comprends tout à fait que l'on soit angoissé par le fait qu'il puisse y avoir un effet moléculaire sans molécules. [...] Il est essentiel que tout cela soit légitimé, cautionné par la communauté scientifique. Seule la publication de nos travaux dans une revue internationale indiscutée permettra d'avancer (9). »

Finalement, le 13 juin 1988, Maddox annonce par téléphone à Benveniste qu'il publie immédiatement l'article en l'accompagnant d'une note d'introduction. Il pose en outre comme condition l'envoi dans le laboratoire de Clamart d'une commission d'enquête après la parution. Au cours de cette longue et tortueuse négociation, Benveniste avait à plusieurs reprises proposé qu'une telle visite ait lieu avant la publication, offre que le rédacteur en chef de Nature avait déclinée.

Ce n'est, en outre, pas la première fois que le chercheur français accède au privilège convoité de publier dans les colonnes de Nature. En 1972, 1977 et 1979, il avait déjà eu l'occasion d'y exposer des travaux relatifs aux effets d'un médiateur important des phénomènes allergiques et inflammatoires connu sous le nom de PAF acéther (10).

L'article paraît donc enfin dans le numéro du 30 juin 1988 de Nature. Il est accompagné d'un éditorial de Maddox intitule : "When to believe the unbelievable ». Ce texte vise à la fois à justifier la publication de résultats aussi étonnants, contraires aux principes fondamentaux de la physique et de la chimie, et à mettre en garde le lecteur qui, par un excès d'enthousiasme, serait amené à les accepter sans recul. Soulignons que le fait d'accompagner un article d'une telle mise en garde est tout à fait exceptionnel. Mais laissons la parole à Maddox : «Des observations inexplicables ne sont pas toujours le signe du surnaturel. Voici ce que les lecteurs du remarquable article de la page 816 doivent garder à l'esprit. [...] Un des buts que doit favoriser la publication de l'article est de fournir un compte rendu authentique de ce travail, au bénéfice de ceux, surtout en France, qui ont accumulé à son propos des rumeurs en provenance de la presse populaire. Un autre est que des membres vigilants de la communauté scientifique, qui possèdent une certaine perspicacité pour mettre en évidence des insuffisances dans le travail des autres, peuvent être capables de suggérer des tests supplémentaires visant à évaluer la validité des conclusions.

(9) Le Monde, dimanche 29-30 mai 1988. C'est nous qui soulignons.

(10) Pour Platelet Activating Factor. 
[...] Les observations de Benveniste, d'autre part, ne sont pas seulement surprenantes parce qu'elles mettent en évidence un nouveau phénomène, mais parce qu'elles heurtent les bases de deux siècles d'observations et d'explications des phénomènes physiques. Que deviendraient par exemple des principes aussi élémentaires que la loi d'action des masses si Benveniste avait raison ? Le principe de restriction qui s'applique ici est simplement que, lorsque des observations inattendues demandent qu'une part substantielle de notre héritage intellectuel soit abandonné, il est prudent de se demander encore plus consciencieusement que d'habitude si ces observations pourraient être incorrectes (11)."

Anticipant de très peu la sortie de l'article dans Nature, le numéro du Monde daté du 30 juin 1988 consacre une partie de sa une à «Une découverte française qui pourrait bouleverser les fondements de la physique » (12). Dans les colonnes du quotidien, Benveniste s'exprime à propos de ses travaux d'une manière qui lui sera par la suite souvent reprochée : "Il s'agit d'entrer dans un autre monde conceptuel. Le changement de mode de pensée n'est pas moins grand que lorsqu'on est passé avec la Terre de la platitude à la rotondité. [...] La procédure utilisée s'apparente à celle qui ferait agiter dans la Seine au Pont-Neuf la clé d'une automobile puis recueillir au Havre quelques gouttes d'eau pour faire démarrer la même automobile, et pas une autre. On comprend dès lors les réticences, voire l'agressivité, au nom de la déesse Raison, des adversaires de ce type d'expériences. [...] Rejeter des résultats étranges alors même qu'ils sont vérifiables sous le prétexte que l' "on n'accepte pas ce que l'on ne comprend pas" serait une attitude rétrograde, antiscientifique, trop répandue chez beaucoup de chercheurs, qui recouvrent leur pusillanimité, leur conformisme, leur stérilité scientifique sous le manteau de la rigueur carté- sienne ; cette situation explique d'ailleurs largement la grande difficulté de la France à faire partie des pays de tête de l'innovation en biologie. [...] Et pour terminer par un rêve, on peut également imaginer que, s'il est possible d'obtenir des entités non moléculaires à partir de molécules, le chemin inverse peut être remonté et qu'un jour l'homme fera des molécules de synthèse à partir des caractéristiques physicochimiques observées à hautes dilutions. Pourra-t-on un jour, de cette façon, transporter instantanément son double électromagnétique à l'autre bout du monde ou dans une autre planète ? Pourra-t-on un jour, à partir de l'information passant sous le Pont-Neuf, reconstituer un diplodocus, ou plus simplement y pêcher un poisson électromagnétique sans arêtes?»

La direction de l'INSERM avait publié le 29 juin 1988 un communiqué de presse soulignant le caractère peu ordinaire des résultats obtenus à Clamart et la nécessité d'un large débat au sein de la communauté scientifique internationale ; ce qui représente une attitude similaire à celle qui est exprimée dans l'éditorial de Nature. Des extraits de ce communiqué figurent dans ce même numéro du Monde. On y souligne le caractère probablement sans précédent dans l'histoire de l'INSERM d'une telle prise de position publique et l'embarras qui l'a sans doute motivée.

Le journal Libération fait également un très large écho à ces découvertes en insistant davantage sur les liens que l'on peut tisser entre celles-ci et l'homéopathie, comme en témoignent les titres des deux numéros successifs : "Une équipe de chercheurs apporte la preuve de l'action des produits homéopathiques » et «La mémoire de l'eau au secours de l'homéopathie » (13). A partir de ce moment, on commence en effet à parler de l'«affaire de la mémoire de l'eau », expression qui va contribuer à renforcer le caractère de «bombe épistémologique et médiatique » de ces travaux en favorisant la proliféra-

(11) Nature $333: 787,30$ juin 1988 . C'est nous qui soulignons.

(12) Le Monde, jeudi 30 juin 1988.

(13) Libération, mercredi 29 juin 1988 et jeudi 30 juin 1988. 
tion de métaphores et de jeux de mots plus ou moins bien intentionnés. Seul Le Monde préférera à cette expression celle plus vague et moins poétique de "mémoire de la matière ».

\section{L'، enquête " de Nature}

La prochaine étape de cette affaire va se dérouler entre le 4 et le 8 juillet 1988, période au cours de laquelle la commission d'enquête imposée par Nature va séjourner à Paris et superviser le déroulement d'un certain nombre d'expériences. La procédure utilisée et la composition de cette commission seront à l'origine d'un surcroît de violence et de passions dans la polémique qui agitait déjà la communauté scientifique et les médias.

Font partie du voyage : John Maddox en personne, Walter Stewart, un scientifique américain qui s'est depuis quelques années spécialisé dans la détection des fraudes scientifiques, James Randi, un illusionniste professionnel souvent employé par des laboratoires s'intéressant à l'étude scientifique des phénomènes paranormaux, et son assistant José Alvarez, lui aussi illusionniste. Stewart est en outre l'un des cinq référents qui ont eu à évaluer l'article de Benveniste ; il était d'ailleurs favorable à la publication.

Au cours de cette expertise, sept expériences vont être réalisées. Les quatre premières donnent des résultats positifs. Pour les trois dernières, le protocole est modifié de manière à permettre la préparation des dilutions et le comptage des basophiles « en aveugle », de manière à minimiser les biais liés à l'observateur (14). A la lumière des résultats obtenus au cours des quatre premières expériences, Jacques Benveniste et Elisabeth Davenas sont très confiants.
Ils se préparent déjà à fêter leur victoire ; la presse a d'ailleurs été convoquée. Mais voici qu'à l'ouverture des codes (15) jusqu'alors tenus secrets les expériences se révèlent négatives. L'expertise, qui avait déjà débuté dans une atmosphère de soupçons, se termine donc dans une ambiance d'extrême tension, pour ne pas dire de psychodrame (16). Maddox va même jusqu'à proposer à Benveniste de se rétracter et de retirer son article.

Le rapport détaillé de la commission d'enquête sera publié dans le numéro de Nature du 28 juillet 1988 (17). Il conclut à la non-existence du phénomène de dégranulation des basophiles. Parmi les très nombreuses critiques qui sont formulées par les trois enquêteurs, voici les principales : un manque de soin dans la pratique des expériences, le caractère difficilement reproductible des résultats, le niveau anormalement bas des erreurs d'échantillonnage, le manque d'efforts pour éliminer les biais statistiques systématiques liés à l'observateur, le climat régnant dans le laboratoire, jugé impropre à l'évaluation objective de résultats exceptionnels. Cette dernière remarque fait surtout référence à la manière dont les membres de l'équipe semblent manifester un désir jugé suspect de « faire marcher les expériences ».

Dans le même numéro de Nature, Benveniste répond aux critiques formulées dans le rapport et s'insurge violemment contre les accusations de fraude et d'incompétence dont il estime être l'objet. Il termine sa plaidoirie de la manière suivante : «J'estime maintenant que ce genre d'enquêtes doit immédiatement être arrêté dans l'ensemble du monde. La chasse aux sorcières de Salem ou des poursuites de type maccarthyste tueront la science. La science ne s'épanouit que dans la liberté.

(14) Dans ce cas, l'expression « en aveugle » signifie que la personne qui effectuait les dilutions ne connaissait pas la concentration de la solution de départ. D'autre part, celle qui comptait les basophiles au microscope n'était pas non plus au courant de la concentration d'anti-IgE introduite dans chaque éprouvette.

(15) Afin d'éviter toute tricherie possible et de manière à pouvoir déterminer le contenu des éprouvettes lors de la lecture des résultats, les tubes à essais furent codés auparavant par les enquêteurs sous la surveillance d'une caméra vidéo. Les codes furent ensuite emballés dans une feuille d'aluminium, puis dans une enveloppe scellée par Randi. Cette dernière fut finalement collée au plafond du laboratoire où elle demeura jusqu'au moment de la lecture finale des résultats.

(16) Pour un compte rendu détaillé des péripéties de l'expertise, comprenant de nombreuses interviews des protagonistes, cf. ALFONSI, 1989, et DE PRACONTAL, 1990.

(17) Nature 334 : 287-290, 28 juillet 1988. 
Nous ne devons à aucun prix laisser la peur, le chantage, la délation, la diffamation et la tromperie s'installer dans nos laboratoires. [...] La seule manière d'établir définitivement l'existence de résultats délicats est de les reproduire. Il se peut que nous nous soyons tous trompés de bonne foi. Ce n'est pas un crime mais de la science habituelle, et seul le futur tranchera (18). » Il réfutera également la plupart des critiques dans une lettre adressée à Science, où il exprimera des opinions semblables (19).

Entre le mois de juillet 1988 et le mois d'août 1989, date à laquelle la revue décide de clore le débat, ce ne sont pas moins de trente-six « lettres » (20) qui seront adressées par des scientifiques de toutes disciplines à Nature. La majorité de ces prises de position vise à déconstruire les résultats obtenus par Benveniste et à fournir des explications alternatives permettant de les réduire à des artefacts non contrôlés ou des biais statistiques. Henri Metzger, l'un des référents consultés par Nature, d'ailleurs totalement opposé à la publication, tentera sans succès de reproduire les résultats.

Dans l'ensemble, on insiste sur le caractère incroyable des faits. La fiabilité du modèle utilisé, le test de dégranulation, employant le sang de patients allergiques, est remise en cause. L'attitude de Nature est parfois critiquée en ce qui concerne sa décision de publier ces travaux et surtout pour son enquête jugée de manière presque unanime comme scandaleuse. Des accusations de collusion avec les laboratoires homéopathiques Boiron sont également formulées. Une minorité de voix, émanant surtout de partisans de l'homéopathie, s'élève pour prendre la défense de Benveniste et de la liberté d'expérimenter, même si le cadre théorique ne s'accorde pas avec les observations.

Dans la grande presse, la visite des « enquêteurs » et leurs conclusions seront relatées avec moult détails. Là aussi, faisant écho à la presse spécialisée, c'est l'étonnement qui prévaut, voire l'indignation face à ce qui est perçu comme de la duplicité de la part de Nature. Dans les colonnes du Monde, Benveniste exprime son indignation dans des termes analogues à ceux employés dans le droit de réponse qui lui a été accordé par Nature (21). Dans un éditorial de Libération, Serge Daney stigmatise « la croyance naïve dans la pureté de la science, croyance qui entraîne une très faible curiosité pour ses procédures réelles » (22). Dans le même numéro, Eric Favereau met en évidence le manque de solidarité manifesté à Benveniste par ses pairs et par son organisme de tutelle, l'INSERM.

D'une manière générale, on constate à partir de l'épisode de l'enquête un revirement, qui se traduit par un certain désintérêt des observateurs pour ce qui touche au contenu de la découverte, à son caractère extraordinaire ou à ses implications éventuelles. Ce changement se fait au profit d'une analyse portant sur les dysfonctionnements des institutions scientifiques et sur l'éventuelle crise dans laquelle ces dernières se trouveraient plongées. Tant en ce qui concerne la prévalence de la fraude, les aléas de l'évaluation, la spécialisation croissante, le conformisme, etc. Ce revirement n'est pas sans liens avec l'entrée en scène de Walter Stewart et de James Randi, qui amènent avec eux un soupçon de fraude et font planer sur la mémoire de l'eau le spectre du paranormal.

\section{La croisade contre l'hérésie}

Arrêtons-nous quelques instants sur le caractère singulier de la contribution de la revue de vulgarisation Science et Vie au débat qui nous occupe. Ses prises de position rationalistes à l'égard de tout ce qui touche aux médecines parallèles ou au paranormal

(18) Nature $334: 291,28$ juillet 1988.

(19) Science $241: 1028,26$ août 1988.

(20) La rubrique «Letters » de Nature comprend aussi bien de simples commentaires que des comptes rendus d'expériences.

(21) Le Monde, mercredi 27 juillet 1988.

(22) Libération, vendredi 29 juillet 1988. 
sont bien connues. Tout au long de la polémique, elle va se distinguer par l'extrême violence de ses propos à l'égard de Jacques Benveniste et de ses partisans. Tous les moyens vont ainsi être mis en œuvre, dans le cadre de quatre numéros, pour discréditer ses résultats. La compétence et l'éthique scientifique de Benveniste sont remises en question. On le soupçonne d'avoir monté un « coup » médiatique avec les laboratoires homéopathiques Boiron (23). Sa paternité en ce qui concerne la découverte du PAF acéther est contestée, ainsi que le nombre et la qualité de ses publications antérieures (24). Il se trouve caricaturé sous les traits d'un illuminé en proie au délire le plus total. Science et Vie fait appel à une pléthore de scientifiques qui viennent tous, à des degrés divers, appuyer ces critiques ou ces accusations.

La revue s'attaque même sans les citer à ses confrères, dont l'ouverture est jugée suspecte : "On souhaiterait aussi savoir pour quelles raisons certains organes de presse ont été si réceptifs à la thèse du docteur Benveniste. Qu'on soit ouvert à des théories audacieuses, c'est certes recommandé, qu'on "gobe" des théories plus proches du mysticisme que de la science dans des publications où l'on compte quand même quelques diplômés de grandes écoles est surprenant (25). »

La revue formule ses propres hypothèses pour rendre compte des résultats obtenus par Benveniste en mettant en cause le colorant utilisé. Elle demande également au professeur Leynardier du Centre d'allergologie de l'Hôpital Rothschild de répéter les expériences. Cette tentative se révèle infructueuse. Elle va même jusqu'à offrir une somme de 1 million de francs français à l'équipe de l'INSERM pour le cas où cette dernière parviendrait à reproduire les résultats dans le laboratoire du professeur Jean Dry, sur la base d'un protocole défini par elle (26).
A deux reprises, faisant écho aux propos de Jacques Benveniste, le fantôme de Lyssenko réapparaît, mais cette fois du côté de la partie adverse. Tout d'abord sous la plume de Denis Buican, un biologiste spécialisé dans l'histoire de la génétique : «Il ne peut s'agir à notre avis que d'une autosuggestion expérimentale collective ou de trucages techniques dignes du lyssenkisme. [...] Si à 1'Est l'affaire Lyssenko l'a prouvé - la science devait servir de béquille au marxismeléninisme, en Occident, la science semble devenue une béquille pour le "Veau d'or" ou pour des superstitions plus ou moins vulgaires (27). »

Un mois plus tard, Gérald Messadié, journaliste, renchérit : «Nous nous sommes laissé dire, en effet, que, dans les milieux qui lui sont favorables, on affirme que "ceux qui ne croient pas à l'homéopathie sont de gens de droite"! C'est le parallèle exact des accusations de Lyssenko : ceux qui ne croyaient pas à sa génétique "marxiste" étaient des "déviationnistes bougeois". Où va se nicher la vertu politique (28)! »

\section{L'évaluation par l'INSERM}

Tous les quatre ans, les unités de recherche de l'INSERM subissent une évaluation qui doit décider de la reconduction de leur mandat. Cette évaluation comprend l'intervention de différentes commissions qui statuent par le biais de rapports, dont les commissions scientifiques spécialisées (CSS) dont dépendent les unités de recherche et le Conseil scientifique, organe faîtier. En dernier ressort, la décision finale de reconduire un directeur de recherche dans son mandat incombe au directeur général de l'INSERM, en l'occurrence Philippe Lazar.

Le mandat de Jacques Benveniste, exmembre du Conseil scientifique de 1982 à 
1986 et de la CSS numéro 1, a débuté en 1980 et devait normalement prendre fin le 30 juin 1992, terme statutaire de ses douze années d'exercice. La CSS numéro 2, dont dépend l'unité 200 , rend son verdict dans un document daté du 25 avril 1989 (29). Tout en reconnaissant la qualité des recherches effectuées dans les domaines du PAF acéther et des autres mécanismes de l'allergie et de l'inflammation, elle émet un avis tout à fait défavorable concernant les recherches sur les hautes dilutions. Elle recommande sur cette base de différer la décision de reconduire Benveniste dans ses fonctions, tant que ce dernier n'aura pas renoncé à ce type de recherches et à « toute relation avec les médias sur les problèmes des hautes dilutions ». Il est évident que les positions adoptées par Benveniste sur la scène publique ont fortement déplu à certains membres de la commission. Elle entend donc laver le préjudice ainsi porté « à l'image de l'INSERM et plus généralement à celle de la communauté scientifique française ", spécialement vis-à-vis de la communauté scientifique anglo-saxonne. Il s'agit donc bien ici d'une sanction.

Le rapport du Conseil scientifique est daté des 4-6 juillet 1989 (30). Pour l'occasion, l'instance d'évaluation s'est exceptionnellement adjoint le concours de deux experts étrangers. Le Conseil évalue globalement l'unité 200 comme «dynamique » et "productive », mais désapprouve les recherches menées sur les hautes dilutions, qui nuisent aux autres travaux et à la réputation du laboratoire. Moins sévère que la CSS numéro 2, il estime que « le renouvellement du mandat de Jacques Benveniste peut être envisagé », mais que «la décision sur ce point devrait cependant être différée, en raison du caractère insuffisamment structuré du programme et des perspectives, et de l'insuffisance des réponses données aux objections graves qui ont été présentées sur certains aspects de l'activité du laboratoire ».

Sans attendre le verdict final du directeur général de l'INSERM, Hubert Curien, ministre de la Recherche et de la Technologie, déclare le 7 juillet sur les ondes d'Europe 1 vouloir « dépassionner cette affaire » et demande «du calme et de la modestie » (31). Il semble prendre la défense de Benveniste en déclarant que, «si l'on ne publie pas des choses dérangeantes, on risque de passer à côté de choses formidables ». Mais il pondère ses propos en soulignant qu' « il ne faudrait pas que s'instaure dans notre pays, comme on l'a vu dans d'autres, l'idée que pour ne pas périr un scientifique doit publier à tout prix, même s'il n'a pas eu le temps de vérifier soigneusement les résultats ».

Le 11 juillet 1989, Philippe Lazar, ayant pris connaissance de ces deux rapports, adresse une lettre à Benveniste (32). Il reconnaît la valeur des recherches effectuées au sein de l'unité 200 . Il se défend, se démarquant ainsi du rapport de la CSS numéro 2 , de vouloir exercer une quelconque censure sur les recherches effectuées dans le domaine de l'effet des hautes dilutions. Il insiste sur « la liberté des chercheurs dans le choix de leurs hypothèses et de leurs modalités de travail » et ne demande pas à Jacques Benveniste « de renoncer à ses idées et aux travaux qui en résultent », car « un tel acte d'autorité contreviendrait gravement au principe de liberté énoncé plus haut ». Il demande simplement à Benveniste de s'efforcer de « rechercher systématiquement les biais expérimentaux qui lui auraient jusqu'à présent échappé et qui peuvent, selon toute vraisemblance, expliquer ses constatations insolites ». Il lui recommande également de « renoncer, pour un temps, à s'exprimer sur ce sujet en dehors des revues scientifiques de haut niveau ».

Sur ces bases plutôt conciliantes, Philippe Lazar décide de surseoir à la confir-

(29) En ce qui concerne le contenu détaillé de ce rapport, cf. ALFONSI, 1989, 299-300, qui donne en annexe de son ouvrage la reproduction de ce document ainsi que celles du rapport du Conseil scientifique et de la lettre de Philippe Lazar, discutés plus bas.

(30) ALFONSI, 1989, 301-305.

(31) Ces propos sont rapportés dans Le Monde, mardi 11 juillet 1989.

(32) ALFONSI, 1989, 307-310. 
mation officielle de la reconduction du mandat de Jacques Benveniste jusqu'au 31 décembre 1989. Dans le courant du mois de janvier, on apprendra finalement qu'il a décidé de reconduire le directeur de l'unité 200 dans ses fonctions pour les trois années à venir. Depuis cette décision, de nouvelles expériences ont été effectuées à Clamart en collaboration avec Alfred Spira, directeur de l'unité 292 de l'INSERM. Les résultats obtenus dans le cadre d'autres protocoles expérimentaux semblent confirmer l'existence d'effets engendrés par de très hautes dilutions de substances (33).

Ici s'achève notre exposé des principales étapes de la controverse. Nous avons montré que cette dernière concerne très largement la définition des modalités de communication et de validation des faits scientifiques. C'est à ces questions que nous allons maintenant nous consacrer.

\section{LA SCIENCE COMME SYSTEME DE COMMUINICATION}

La circulation de l'information dans le champ scientifique a constitué de longue date un objet d'étude majeure pour la sociologie des sciences. Chez Merton, déjà, les rapports entre communication et validation des connaissances occupent une place centrale. Les quatre normes qui selon lui s'inscrivent au cœur de la pratique scientifique - l'universalisme, le désintéressement, le scepticisme organisé et le « communisme »- ont pour corollaire la mise en commun des produits de l'activité des chercheurs. "La conception institutionnelle de la science comme faisant partie du domaine public est reliée à l'impératif de communication des résultats. Le secret est l'antithèse de cette norme ; la communication complète et ouverte, sa réalisation. La pression pour la diffusion des résultats est renforcée par l'objectif institutionnel de repousser les frontières de la connaissance et par l'incitation à la reconnaissance qui dépend naturellement de la publication (34). »Par « domaine public » il faut naturellement entendre ici le groupe des seuls pairs qui peuvent de manière légitime prétendre à la connaissance des résultats et à leur évaluation.

Les interactions multiples et contestées qui s'opèrent entre la littérature « sacrée » et la littérature «profane » (35) dans l'affaire des hautes dilutions nous renvoient à cette aube de la science expérimentale qui voit se tracer les limites entre le réseau chargé d'assurer l'expansion et la circulation des faits scientifiques et le reste de la société.

Le problème posé à la communauté scientifique naissante, qui s'est traduit au XVII ${ }^{e}$ siècle par la création de la Royal Society britannique et des premières revues scientifiques, peut être formulé de la manière suivante : comment mettre en place un système de communication qui assure tout à la fois la circulation et la validation de l'information par la formation d'un consensus portant sur les faits scientifiques entre les membres de la communauté des savants ? C'est aux expérimentalistes anglais du milieu du XVII ${ }^{e}$ siècle, et en particulier à Robert Boyle, l'inventeur de la pompe à vide, qu'il faut attribuer la mise en place d'une configuration socio-technique susceptible de construire et de communiquer des « faits » scientifiques au sens où nous l'entendons aujourd'hui. Ce qui pour l'observateur de la science contemporaine semble aller de soit relève bel et bien de pratiques élaborées entre la fin des années 1650 et le début des années 1660 concernant la mise en évidence de l'existence et des propriétés du vide. Comme le souligne Shapin (36), la création des faits nécessita la mise en œuvre coordonnée de trois " technologies" indissociables : une technologie matérielle (les instruments), une technologie sociale (les normes et conventions élaborées au

(33) BENVENISTE et al., Comptes rendus de l'Académie des sciences, Paris 312 (11), 461-466, 1991.

(34) MERTON, 1973 a, 274.

(35) Sur cette distinction et ses implications sociologiques, cf. JURDANT, 1973.

(36) SHAPIN, 1990. Pour des développements plus approfondis concernant ces questions, cf. SHAPIN et SCHAFFER, 1985. 
sein de la communauté des philosophes de la nature pour évaluer les revendications de connaissance) et une technologie littéraire.

La circulation de l'information scientifique repose, dès l'origine, essentiellement sur les revues spécialisées dont les précurseurs furent, en France, le Journal des Sçavants, fondé en janvier 1665 , et The Philosophical Transactions, revue de la Royal Society établie en mars de la même année. Parallèlement à la création de ces supports, on met en place la fonction d'éditeur et le système des référents qui est censé assurer la qualité des travaux publiés (37). Dès le début, ce dispositif assurera l'économie de la propriété intellectuelle des découvertes, ainsi que leur validation dans le champ scientifique.

Cette parenthèse historique nous semble nécessaire dans la mesure où elle situe précisément le lieu d'émergence de problèmes qui, dans le champ scientifique contemporain, se trouvent au centre de vastes controverses. Il s'agit principalement de la question de l'évaluation par les pairs et du fonctionnement des comités de lecture des revues scientifiques. Il s'agit plus généralement des modalités de circulation de l'information scientifique, c'està-dire des moyens à mettre en œuvre pour assurer la qualité des produits du champ scientifique, et de l'étendue qu'il convient de donner à leur diffusion. Les revues scientifiques spécialisées occupent une place centrale dans ce débat, et c'est à l'examen de leur contribution que nous voulons maintenant nous consacrer.

\section{La littérature sacrée}

La controverse sur l'effet des hautes dilutions permet d'analyser le rôle joué par les revues dans la construction des faits scientifiques. En dernière instance, c'est en leur sein que les produits de l'activité scientifique sont présentés, évalués et critiqués. Elles constituent donc le champ de bataille privilégié, mais pas exclusif, au sein duquel se déploient les controverses scientifiques. Nous commencerons par décrire brièvement le fonctionnement d'une revue comme Nature (38). Nous aborderons ensuite les questions soulevées par le comportement de cette dernière dans l'affaire qui nous occupe. Cela nous permettra de discuter de quelques problèmes qui se posent dans le cadre de l'évaluation de la recherche par le biais des publications scientifiques (39).

Une revue se caractérise tout d'abord par le public qu'elle désire atteindre, le type d'articles qui y sont publiés et le niveau de ceux-ci. Ces différents paramètres peuvent être regroupés sous le terme général de «politique éditoriale». La revue Nature s'adresse à un vaste public de chercheurs de toutes disciplines. Il s'agit d'une revue destinée à des professionnels de la science, mais néanmoins généraliste, dans la mesure où elle traite aussi bien de physique et de biologie que de géologie. Outre les articles scientifiques proprement dits, on y trouve de nombreux articles généraux d'information et d'opinion, qui abordent tous les sujets touchant aux sciences et aux techniques. La seule revue qui lui soit véritablement comparable est sa consœur américaine Science, qui jouit d'une réputation analogue.

Nature, en près de cent vingt ans d'existence, a acquis la réputation de publier des travaux d'avant-garde. Citons par exemple la découverte de la structure de l'ADN (1953), la confirmation de la dérive des continents (1962), la découverte des pulsars (1971) ou la structure du virus du sida (1985). Il est inutile d'insister sur le fait que, pour un chercheur, la possibilité d'accéder à la publication dans une telle

(37) Sur la mise en place du système des référents, cf. MERTON, 1973 b.

(38) Outre la pertinence de cette brève description pour la compréhension de notre propos, notons, fait symptomatique, que l'ouvrage dont nous avons tiré certains éléments concernant ce chapite (HUGUIER et MAISONNEUVE, 1990), un guide de rédaction à l'usage des scientifiques, mentionne l'affaire de la mémoire de l'eau afin d'illustrer les difficultés rencontrées par les comités de rédaction dans le traitement des articles. On ne peut qu'espérer que les analyses du discours scientifique, et plus généralement les recherches sur le rôle des inscriptions dans la production du savoir, s'étendent à ce genre de guides, ainsi qu'aux normes édictées par les revues.

(39) Pour une discussion des différentes approches de la scientométrie, cf. COURTIAL, 1990. 
revue constitue un gage quasi automatique de reconnaissance.

Chaque semaine, le comité de rédaction reçoit environ 150 articles, et ce nombre est en constante augmentation. Le comité ne peut à lui seul évaluer l'ensemble de ces travaux. Il a donc recours à un certain nombre d'experts extérieurs : les référents. Le carnet d'adresses de Nature en compte environ 7000 . Le comité de rédaction va donc envoyer les articles à expertiser aux spécialistes de la discipline concernée. Plusieurs questions importantes se posent à ce niveau. Il s'agit tout d'abord du problème de l'indépendance de jugement du comité par rapport aux référents. Un autre point délicat, actuellement vivement débattu dans les milieux spécialisés aux Etats-Unis, est celui du caractère anonyme de l'expertise qui, en principe, devrait être le garant de l'impartialité et de la sérénité des débats. Certains comités de rédaction exigent même un double anonymat, le nom des auteurs et celui de l'institution où le travail a été réalisé demeurant cachés aux référents. D'autres revues, comme $\mathrm{Na}$ ture, acceptent une expertise nominale et demandent même parfois aux auteurs de leur suggérer le nom d'un ou de plusieurs référents. Il est rare que plus de trois référents soient consultés concernant un même article. Cependant, certains cas particulièrement difficiles peuvent nécessiter la collaboration de davantage d'experts, comme ce fut le cas pour l'article sur l'effet des hautes dilutions, qui nécessita l'examen contradictoire de cinq personnes.

Une fois en possession des expertises, le comité de rédaction prend une décision vis-à-vis du manuscrit soumis à la revue : acceptation immédiate et sans révision, refus ou suggestion de modifications aboutissant à une nouvelle soumission du manuscrit. Les modifications peuvent concerner des aspects de détail ou demander une ou plusieurs séries d'expérimentations complémentaires. Ces aller-retour entre le comité de rédaction, les référents et les auteurs peuvent se répéter plusieurs fois. La complexité du processus de traitement d'un article explique que le délai de publication atteigne couramment dix à douze mois et que la durée entre l'acceptation définitive du manuscrit par le comité de rédaction et sa publication ne soit inférieure à quatre mois que dans les meilleures conditions. Il convient en outre de remarquer, que pour des revues très sélectives comme Nature ou Science, une proportion très importante d'articles sont retournés à leurs auteurs avant même d'avoir été soumis à un référent.

Dans le domaine de la recherche biomédicale, on compte actuellement plusieurs milliers de périodiques. Pour un scientifique désireux de publier les résultats de ses travaux, la première question qui se pose réside donc dans le choix d'une revue. L'audience et le crédit qu'un auteur est en droit d'attendre de ses pairs dépendent en grande partie de ce choix : "L'importance de l'article de Benveniste et collaborateurs ne réside pas dans les résultats de l'étude eux-mêmes, mais dans le fait qu'il a été publié dans Nature, plutôt que dans le British Homeopathic Journal. Des revues d'homéopathie et autres journaux ont depuis des années publié des résultats d'expériences démontrant l'activité de très hautes dilutions. Beaucoup de ces études ont été menées d'une manière scientifiquement rigoureuse (40). »

Cette déclaration est appuyée par celle d'un scientifique adversaire de l'homéopathie : «Cela se révélera être encore un autre cas d'artefact (ou pire), mais le mal a déjà été fait ; la reconnaissance générale de cet important paradigme de l'homéopathie par une revue scientifique de premier plan (41). » C'est donc l'importance stratégique de la publication dans Nature de travaux qui semblent confirmer les principes thérapeutiques de l'homéopathie qui est à l'origine d'une première série de critiques adressées au comité de rédaction de la revue. C'est dans ce sens qu'il faut interpréter le rôle que jouent les revues scientifiques dans la perpétuation de ce que Pierre 
Bourdieu nomme «l'ordre scientifique établi », rôle auquel Nature ne déroge ici qu'en apparence : «Outre les instances spécifiquement chargées de la consécration (académies, prix, etc.), [l'ordre scientifique établi] comprend aussi les instruments de diffusion, et en particulier les revues scientifiques qui, par la sélection qu'elles opèrent en fonction de critères dominants, consacrent les productions conformes aux principes de la science officielle, offrant ainsi continûment l'exemple de ce qui mérite le nom de science, et exercent une censure de fait sur les productions hérétiques soit en les rejetant expressément, soit en décourageant purement l'intention de publication par la définition du publiable qu'elles proposent (42).»

Cette fonction normative, Nature semble l'assumer d'une manière particulièrement originale. Ce n'est en effet pas la première fois que la revue prend le risque de publier des travaux présentant des résultats très controversés, pour ne pas dire hérétiques. En 1972, elle publia un article qui prétendait démontrer la possibilité de transmettre un comportement appris par les rats d'un premier groupe à un second groupe de rats. Ce comportement consistait en un conditionnement aversif vis-àvis de l'obscurité. La transmission était opérée par l'injection d'une protéine, appelée scotophobine, prélevée chez les rats du premier groupe, dans le cerveau des rats du second groupe (43). Lors de sa publication, l'article fut accompagné d'un commentaire dévastateur de Walter Stewart, qui débuta ainsi sa carrière de «chasseur de fraudes ». En 1974, Nature publia un article qui semblait mettre en évidence les pouvoirs de perception extrasensorielle du médium Uri Geller par deux physiciens américains de l'université californienne de Stanford (44). Dans ce cas, l'article était accompagné par un éditorial comportant des commentaires sur la posi- tion de l'éditeur et par des extraits de rapports de lecture défavorables (45). Le rôle d'une revue scientifique se limite généralement à l'examen attentif des articles qui lui sont soumis pour publication et ne comprend pas la falsification ou la répétition d'expériences jugées douteuses. Pourquoi donc décide-t-elle de donner audience à de tels travaux ?

Les réponses du rédacteur en chef de Nature à ses détracteurs illustrent que, par son comportement dans l'affaire de la mémoire de l'eau, la revue n'a pas dérogé à son rôle de "gardien du temple de la science », comme pourrait le laisser croire sa décision de publier des résultats hétérodoxes. Dans une réponse adressée à Arnold Relman, éditeur de la célèbre revue concurrente New England Journal of Medicine, Maddox déclare : «Je pense que nos lecteurs auront été instruits sur trois points importants par l'article de Benveniste et ses séquelles : comment la science authentique peut être facilement imitée par la sélection soigneuse des données et l'usage judicieux du langage ; comment des articles, même expertisés avec rigueur et honnêteté, peuvent comporter des défauts identifiables même par des gens que Benveniste (avec raison, dans le contexte) nomme « amateurs », et - de manière plus alarmante - à quel point il est vraisemblable que beaucoup de science de second niveau trouve son chemin vers la publication (46). »

Dans un article du New York Herald Tribune daté du 26 septembre 1988 repris par la revue Esprit, on peut lire, toujours sous la plume de John Maddox : "Tout le monde salue la science de haut niveau et ses prix Nobel. Mais qui vous parle d'une science de second niveau, et qui vous dit où la trouver? [...] Celle-ci n'est jamais discutée en raison même de la conviction qu'il n'y a de science que parfaite. [...] La critique est en réalité confinée dans les

(42) BOURDIEU, 1976, 96.

(43) UNGAR et al., Nature $238: 198-202,1572$.

(44) TARG et PUTHOFF, Nature $251: 602-607,1974$

(45) Il en est donc parfois des textes scientifiques comme des classiques sur le scriptorium des scolastiques : le commentaire devient ce paratexte qui canalise la « juste interprétation » qui peut aller jusqu'à la dénégation.

(46) Science $241: 1586,23$ septembre 1988. C'est nous qui soulignons. 
propos privés, les discussions de séminaire, les rapports privés ou les conversations d'après-repas. Cela permet à la science de second niveau d'être épargnée par les coups de tambour quotidiens du scepticisme. [...] Il faut reconnaître son existence et la mettre en discussion. La science de haut niveau serait d'autant plus garantie et il y aurait moins de confusion dans l'esprit public concernant la signification et le rôle de la science (47). »

S'il est bien difficile de savoir ce que Maddox entend par «science de second niveau », on peut constater que Nature a adopté une politique éditoriale particulière, qui consiste à accepter de publier des travaux hétérodoxes dans le but de mettre en évidence leurs lacunes ou de stigmatiser leurs auteurs. La revue entend ainsi occuper une position de leader dans la définition des normes de l'excellence scientifique, par le biais de stratégies dont le moins que l'on puisse dire est qu'elles ne font pas l'unanimité. Ce rôle peut d'ailleurs également être mis en évidence dans le cas des travaux consacrés à Uri Geller et aux effets de la scotophobine.

\section{La littérature profane}

Par de nombreux aspects, l'affaire de la mémoire de l'eau ne représente pas un objet d'étude habituel pour la sociologie des sciences. En effet, dans cette controverse, les affrontements se déroulent non seulement par l'intermédiaire des périodiques scientifiques et au sein des milieux spécialisés, mais aussi sur le terrain plus large des revues de vulgarisation et de la grande presse. L'usage croissant que les scientifiques font des médias ou de ce que Michel Callon, à la suite de Nature, nomme " la science par conférence de presse » (48) représente une tendance nouvelle et révélatrice du fonctionnement du champ scientifique contemporain (49). Cette évo- lution trouve son illustration dans plusieurs découvertes récentes comme la supraconductivité à haute température, la « fusion froide » ou l'isolement du virus du sida, dans lesquelles l'effet d'annonce a joué un rôle important. Mais c'est sans doute dans le cadre de l'affaire de la mémoire de l'eau que l'importance stratégique que les médias peuvent jouer dans la circulation des faits scientifiques est illustrée avec le plus de clarté.

Comme nous l'avons mis en évidence, les effets des hautes dilutions ne sont pas seulement, de manière simultanée, construits et déconstruits au sein de la littérature spécialisée, mais aussi sur la scène publique, par l'intermédiaire du Monde, de Libération ou de Science et Vie. Parmi les différentes ressources dont disposent les scientifiques pour enrôler de nouveaux alliés (50), les médias constituent une alternative importante.

Face à l'inertie et au conformisme de certaines institutions, organismes de tutelle ou revues scientifiques, les chercheurs peuvent être tentés de porter sur la scène publique le débat généralement confiné au sein de la communauté scientifique.

Pour la plupart des chercheurs, une telle démarche constitue une violation impardonnable de la déontologie et de la méthode scientifique. Une grande partie de la polémique porte en effet sur le caractère public de l'information et sur l'effet d'annonce ainsi provoqué. Pour ses pairs, Jacques Benveniste est avant tout coupable d'avoir donné une large audience à une controverse que beaucoup de scientifiques et, en particulier, ses supérieurs hiérarchiques auraient préféré voir confinée dans les revues spécialisées et les laboratoires. Rappelons ici les recommandations tout à fait significatives faites au directeur de l'unité 200 par Philippe Lazar : « La ligne de conduite que je vous recommande suppose en particulier que vous renonciez,

(47) MADDOX, 1988, 106-108. C'est nous qui soulignons.

(48) CALLON, 1990.

(49) Il serait intéressant de s'interroger sur les parallèles que l'on peut tirer entre le recours aux médias dans ce contexte et les usages qu'en font les spécialistes des sciences humaines dans les rubriques qui leur sont réservées dans la presse.

(50) Sur ces concepts, cf. LATOUR, 1989 a. 
pour un temps, à vous exprimer sur ce sujet en dehors des revues scientifiques de haut niveau - le temps nécessaire pour que vous puissiez reconstituer le capital de confiance que vous avez aujourd'hui, que vous l'admettiez ou non, en grande partie dissipé aux yeux de vos collègues (51). »

Les « manquements » de plus en plus fréquents qu'il nous est donné d'observer à la règle consistant à ne pas divulguer dans l'espace public des résultats de recherche qui ne sont pas marqués du sceau d'un consensus suffisant éclairent sous un jour nouveau l'évolution de l'espace de communication du champ scientifique. Parfois, c'est dans le but de se prémunir contre les possibilités d'une «fuite » avant publication que les chercheurs ont recours à des annonces préliminaires sous la forme de conférences de presse. Nous retrouvons ici l'importance accordée dans le champ scientifique à la notion de priorité. Sur un plan plus général, c'est la nécessité d'augmenter de manière exponentielle la visibilité de leur travaux qui conduit les chercheurs à avoir recours aux médias. Cette nécessité réside dans l'obligation où ils se trouvent d'intéresser d'autres alliés potentiels (personnes, industries, institutions, fondations) à leur produits, alliés dont le recrutement par le biais des canaux habituels de publication pourrait s'avérer difficile, voire impossible. Les scientifiques tendent donc, face à la rigidité de l'organisation des institutions de recherche, à mettre en place des « réseaux de coopération flexibles » (52) susceptibles d'accroître leur crédit. En effet, les clivages disciplinaires, les « chapelles » de toutes sortes qui concentrent la légitimité scientifique ainsi que le pouvoir et le monopole exercés par les revues et leurs référents dans la diffusion des connaissances rendent l'émergence de certains types de résultats ou programmes de recherche pratiquement impossible.

La compétition croissante entre les laboratoires, qui se traduit principalement par la nécessité de publier à tout prix «publish or perish »- et de décrocher sans cesse de nouvelles subventions met en évidence les écarts fonctionnels par rapport à l'idéal de communication hérité des sociétés savantes du XVII ${ }^{e}$ siècle. La dialectique diffusion-rétention d'informations se trouve confrontée à de nouvelles contraintes qui débouchent sur le fait que " chaque réseau s'invente un équilibre entre la création d'un espace public de discussion et un espace privé, entre la divulgation auprès des quelques concurrents, la protection par les brevets et l'annonce faite aux médias. Aucun critère universel ne permet d'établir ces frontières. Leur tracé dépend de considérations stratégiques et de l'état des forces en présence (53) ».

Dans ce contexte, les médias constituent des vecteurs permettant de jeter des ponts par-dessus les infrastructures de recherche et de publication jugées trop lourdes, voire inaccessibles. De cette manière, les chercheurs ont la possibilité de mettre en place de nouveaux réseaux très étendus qui peuvent crôtre rapidement en intégrant des alliés qui autrement seraient probablement demeurés en dehors de leur sphère de recrutement. Les médias constituent donc ce que nous nommerons des « amplificateurs de réseaux ». La stratégie adoptée par Benveniste est en ce sens exemplaire, dans la mesure où John Maddox reconnaît que sa décision de publier ces résultats « incroyables " a été motivée par la publicité qui leur avait été faite auparavant dans les colonnes du journal Le Monde (54).

D'autres déclarations du chercheur français, qui comparent l'importance de ses résultats et sa situation à celles de Galilée et de Copernic, stigmatisent les méfaits de "l'inquisition » et de la «chasse aux sorcières » dont sont victimes les thèses « hérétiques », doivent également être interprétées dans ce sens. Les ressources que Benveniste mobilise ici grâce aux médias sont celles que lui offre l'histoire des sciences. Cette stratégie est en effet rendue

(51) Lettre de P. Lazar rapportée in ALFONSI, 1989, 309.

(52) Nous empruntons cette expression à CALLON, 1990.

(53) CALLON, 1991 b, 440.

(54) Nature $333: 787,30$ June 1988. 
plus transparente si l'on considère, comme l'a bien montré Isabelle Stengers, que la « rationalité » n'est pas un attribut de la pratique scientifique, mais un enjeu. Les négociations et les luttes entre les agents du champ scientifique qui visent à délimiter le domaine de la science et de la méthode scientifique légitimes, passent en partie par une « reconstruction » de l'histoire des sciences (55).

Par la formulation d'un récit, souvent épique, mettant en scène la genèse de leur discipline, les scientifiques balisent ainsi la route qui mène à leur champ d'investigation présent, traçant dans le même mouvement la frontière entre problématiques pertinentes et dépourvues d'intérêt, entre approches orthodoxes et hétérodoxes. Par ses déclarations tonitruantes, Benveniste s'institue comme le Galilée de la biologie moderne, luttant contre les forces obscurantistes d'institutions sclérosées et conservatrices. La crédibilité de ses fragiles résultats se trouve ainsi renforcée, au moins provisoirement. Lorsqu'il généralise la portée de ses expériences dans les colonnes du Monde, en formulant des hypothèses qui semblent sortir tout droit d'un ouvrage de science-fiction, ce n'est rien moins que les ressources de l'imaginaire collectif qu'il tente de mobiliser en sa faveur.

\section{Les forums de l'information scientifique}

L'affaire de la mémoire de l'eau présente de nombreux points communs avec ce que Pinch et Collins ont mis en évidence dans le cadre de travaux portant sur les controverses à propos de la recherche sur les phénomènes paranormaux aux Etats-Unis. Les parallèles que nous pouvons faire entre ces deux situations sont intéressants, car rares sont les sociologues qui se sont attachés à étudier dans les controverses scientifiques contemporaines l'interaction entre les circuits de l'information scientifique légitime et ceux de l'espace public mass-médiatique. Ces auteurs ont ainsi été amenés à distinguer un forum constituant «qui comprend la théorisation scientifique et l'expérimentation ainsi que la publication et la critique correspondantes dans les revues spécialisées » et un forum officieux « dans lequel nous trouvons les articles des revues populaires ou semi-populaires, les discussions et les ragots, les actions visant à réunir des fonds ou à se faire de la publicité » (56).

Dans le cas de la parapsychologie comme dans celui des hautes dilutions, nous observons une contamination à l'intérieur des deux forums entre les arguments « scientifiques» et les arguments « non scientifiques ». Dans les deux cas, il nous est donné d'observer un rejet a priori basé sur un préjugé d'impossibilité, alors même que les protocoles expérimentaux sont le plus souvent au moins aussi rigoureux que dans la moyenne des expérimentations menées en laboratoire. Dans les deux situations, on observe un refus d'entrer en matière prétextant un fondement théorique insuffisant des phénomènes extraordinaires mis en évidence. Le soupçon de fraude se trouve à plusieurs reprises au centre de la discussion et s'associe à un certain nombre d'attaques touchant à la personnalité des protagonistes. La mention de l'origine des fonds ayant financé les recherches est utilisée pour discréditer les résultats.

Dans les deux situations, « l'antagonisme scientifique envers le nouveau phénomène est assez fort pour que les scientifiques et les philosophes acceptent même des outsiders non scientifiques comme alliés afin de maintenir les conceptions courantes » (57). Les journalistes de Science et Vie organisent une expérimentation contradictoire et offrent 1 million de francs

(55) Sur les usages stratégiques de l'histoire des sciences, cf. STENGERS, 1986. Dans une même perspective, on pourra également consulter l'article consacré par le même auteur aux différentes interprétations de « l'affaire Galilée » dans STENGERS, 1989. En ce qui concerne les implications de ce problème pour le sociologue et l'historien des sciences, cf. LATOUR, $1989 \mathrm{~b}$.

(56) COLLINS et PINCH, 1990, 302.

(57) COLLINS et PINCH, 1982, 156. 
de récompense à qui parviendra à apporter la preuve de l' « incroyable ». Comme dans les expériences de parapsychologie auxquelles il se trouva intimement lié, Randi met en cuvre une variante originale des thèses de Popper : la falsification par l'illusion. Etant donné le degré d'hérésie des résultats présentés, tous les coups sont permis et le terme irénique de « communauté scientifique » paraît bien dérisoire devant la violence et le caractère passionnel des arguments avancés par les différents protagonistes.

L'hêtérogénéité des ressources mobilisées par les acteurs dans les différents forums peut prendre au dépourvu les conceptions «classiques » de la philosophie et de l'histoire des sciences. Cette situation doit nous inciter à substituer à une approche qui différencie clairement contenu et contexte, intérieur et extérieur des pratiques scientifiques, un modèle qui rende compte de l'ensemble des médiations qui permettent la production et la circulation des faits scientifiques.

\section{Les réseaux communiquants de la science}

La géométrie variable des frontières de l'espace public susceptible d'assurer la communication et la validation des faits scientifiques ne saurait surprendre que ceux pour qui la science et ses praticiens disposeraient d'un espace de production étanche : celui du laboratoire. Or, comme l'ont bien montré les récents travaux de sociologie et d'anthropologie des sciences et des techniques, ce lieu mythique n'acquiert et ne maintient ses propriétés de producteur de faits scientifiques que lorsqu'il parvient à s'inscrire au sein d'un vaste réseau de médiations socio-techniques.

Par réseau (58), il faut entendre ici l'ensemble des éléments humains et non humains - molécules, cellules, animaux, ins- truments, chercheurs, institutions, crédits, théories, conceptions épistémologiques et textes de toutes sortes - qui le composent. Certes, le laboratoire occupe dans ce dispositif une place centrale, puisqu'il capitalise et transforme en son sein l'ensemble des ressources mobilisées par le biais du réseau. Les textes, supports de l'information scientifique, occupent une place essentielle dans le processus de circulation et de construction des faits. Les articles des revues spécialisées ne représentent, comme le fait remarquer Latour (59), que le sommet de l'iceberg d'une longue chaîne de transformations textuelles opérées par les chercheurs qui comprend également prépublications (preprints), télécopies, courrier électronique, demandes de crédit, lettres de recommandations, ouvrages et articles de vulgarisation, etc.

L'affaire de la mémoire de l'eau illustre un aspect trop souvent ignoré par la philosophie et l'histoire des sciences : l'intérêt que suscite l'émergence d'un fait scientifique n'a rien d'immanent ; il résulte d'un intéressement réussi de la part de ceux qui désirent l'établir et le communiquer. Divulger un fait scientifique nouveau et organiser les débouchés pour en permettre la circulation participent d'une même opération. Le scientifique est aussi un entrepreneur qui, pour intéresser les autres, doit mobiliser et agencer un ensemble de ressources hétérogènes (60). Les médias, en tant qu'amplificateurs de réseaux, constituent l'une de ces ressources. Par leur intermédiaire, les expériences sur les hautes dilutions deviennent tour à tour susceptibles de bouleverser les lois de la physico-chimie, fondements scientifiques pour l'homéopathie, révélatrices des dysfonctionnements du champ scientifique, honte de la communauté scientifique française, source d'investissements imaginaires dans une science que l'on croyait désenchantée, occasion d'une relecture de l'histoire des sciences, revanche de la

(58) Nous nous référons ici à une conception des réseaux socio-techniques développée dans le cadre du modèle de la «traduction » par Latour et Callon. Pour un exposé détaillé de ce modèle et de ses implications pour notre projet de reconstruction des médiations, cf. CALLON, 1991 a et VINCK, 1991.

(59) LATOUR, 1991.

(60) Pour une analyse de l'activité scientifique décrite en ces termes cf. LAW, 1986. 
science légère sur la science lourde, etc.

Ces différentes versions ne représentent que quelques-unes des nombreuses traductions (61) que les textes font subir au phénomène de la mémoire de l'eau, le faisant passer dans le même mouvement du fait à l'artefact, du rationnel à l'irrationnel, de la vérité à la fausseté. Comme dans le cas analysé par Collins et Pinch, dans l'affaire de la fusion froide ou dans celle de la découverte du virus du sida, les arguments utilisés dans les deux forums de l'information scientifique se superposent à un tel point qu'il serait bien téméraire de vouloir en tracer les frontières. Ce brouillage n'effrayera en rien celui qui reconnaît dans ce foisonnement de traductions contradictoires la manifestation amplifiée de l'activité quotidienne des scientifiques-entrepreneurs, préoccupés par la croissance de leurs réseaux.

La situation qu'il nous est donné d'observer présente toutes les apparences du paradoxe : c'est au moment où l'omniprésence des sciences semble atteindre son apogée que les formes de coordination traditionnelles du travail scientifique qui en ont assuré l'émergence trouvent leur limite. Le recours croissant des scientifiques à des modes de communication alternatifs participe peut-être d'une tentative de restaurer un équilibre entre une nécessaire autonomie et les contraintes d'un marché du financement de la recherche et de l'innovation en pleine mutation. Les médias et les ouvrages de vulgarisation offrent aux chercheurs un espace affranchi des normes canoniques imposées par les publications scientifiques. Les prises de position historiques, politiques ou épistémologiques qui n'ont pas ou plus leur place dans la littérature sacrée peuvent se déployer dans la littérature profane. On aurait tort, nous l'avons illustré, de trop insister sur les différences entre ces deux types d'écrits qui participent à la définition des règles du jeu. Nous ne suivrons pas les esprits chagrins qui ne perçoivent dans les affaires récentes, la prolifération de la littérature de vulgarisation ou le recours aux médias, que les marques de la déchéance d'un paradis perdu de la cité scientifique. Ce que le champ scientifique semble perdre en autonomie et en "pureté », il le gagne en visibilité. Cette transformation devrait satisfaire à la fois l'analyste et le citoyen.

Si l'affaire de la mémoire de l'eau met à jour, dans une certaine mesure, les lacunes ou les hésitations des circuits de l'information scientifique légitime, son analyse peut contribuer à l'élaboration d'une sociologie globale de la communication scientifique. Elle nous incite à suivre, sans sombrer dans le relativisme radical, les multiples chemins qui mènent à la construction des faits scientifiques qui, pour reprendre les termes de Jacques Benveniste, «ne naissent pas tous égaux en droit » (62). 
ALFONSI P., Au nom de la Science, Paris, Barrault-Taxi, 1989.

BENVENISTE J., « La Vérité scientifique : faux vrai passeport pour de vraies frontières ", Traverses (47), 58-67, novembre 1989.

BOURDIEU P., « Le Champ scientifique », Actes de la recherche en sciences sociales (2-3), 88-104, 1976.

CALLON M., « La Science par conférence de presse ", La Recherche 21, 1184-1190, octobre 1990.

CALLON M., « Réseaux technico-économiques et irréversibilités », in Boyer R., Chavance B. et Godard O. (éd.), Les Figures de l'irréversibilité en économie, 195-230, Paris, Editions de l'Ecole des hautes études en sciences sociales, 1991 a.

CALLON M., « La Recherche en quête de nouveaux modes d'organisation », in Witkowski N. (éd.), L'État des sciences et des techniques, 438-441, Paris, La Découverte, $1991 \mathrm{~b}$.

COLLINS H.M et PINCH T.J., Frames of Meaning. The Social Construction of Extraordinary Science, London, Routledge and Kegan Paul, 1982.

COLLINS H.M et PINCH T.J., « En parapsychologie, rien ne se passe qui ne soit scientifique... », in Callon M. et Latour B. (éd.), La Science telle qu'elle se fait, 297343, Paris, La Découverte, 1991.

COURTIAL J.-P., Introduction à la scientométrie, Paris, Anthropos, 1990.
DAVENAS E., BEAUVAIS F., AMARA J., OBERBAUM M., ROBINZON B., MIADONNA A, TEDESCHI A., POMERANZ B., FORTNER P., BELON P., SAINTE-LAUDI J., POITEVIN B. et BENVENISTE J., « Human basophil degranulation triggered by very dilute antiserum against $\operatorname{IgE} »$, Nature 333, 816-818, $30^{\text {rd }}$ june 1988.

DE PRACONTAL M., Les Mystères de la mémoire de l'eau, Paris, La Découverte, 1990.

HUGUIER M. et MAISONNEUVE H., La Rédaction médicale, Paris, Doin, 1990.

JURDANT B., Les Problèmes théoriques de la vulgarisation scientifique, Thèse de doctorat de troisième cycle, Strasbourg, Université Louis-Pasteur, 1973.

LATOUR B., La Science en action, Paris, La Découverte, 1989 a.

LATOUR B., « Pasteur et Pouchet : hétérogenèse de l'histoire des sciences ", in Serres M. (éd), Eléments d'histoire des sciences, 423-445, Paris, Seuil, 1989 b.

LATOUR B., « Le Royaume de l'écrit scientifique », in Witkowski N., (éd.), L'Etat des sciences et des techniques, 441443, Paris, La Découverte, 1991.

LAW J., "Laboratories and Texts », in Callon M., Law J. and Rip A. (éd), Mapping the Dynamics of Science and Technology, London, Macmillan, 1986.

MADDOX J., «Les Scientifiques ne sont pas tous des prix Nobel », Esprit, 106-108, novembre 1988. 
MERTON R.K., "The Normative Structure of Science », in Merton R.K., The Sociology of Science. Theoretical and Empirical Investigations, 267-278, Chicago, The University of Chicago Press, 1973 a.

MERTON R.K., « Institutionalized Patterns of Evaluation in Science », in Merton R.K., The Sociology of Science. Theoretical and Empirical Investigations, 460-496, Chicago, The University of Chicago Press, 1973 b.

SHAPIN S., «Une Pompe de circonstance. La technologie littéraire de Boyle », in Callon M. et Latour B. (éd.), La Science telle qu'elle se fait, 37-86, Paris, La Découverte, 1991.

SHAPIN S. et SCHAFFER S., Leviathan and the air-pump. Hobbes, Boyle, and the experimental life, Princeton, Princeton University Press, 1985.
STENGERS I., «L'Histoire des sciences et comment s'en servir », in Dupuy J.-P. et al., Sens et place des connaissances dans la société, 117-145, Paris, Editions du CNRS, 1986.

STENGERS I., « Les Affaires Galilée », in Serres M. (éd.), Eléments d'histoire des sciences, 223-249, Paris, Bordas, 1989.

VINCK D., La Coordination du travail scientifique. Etude de deux formes spécifiques: le laboratoire et le réseau, Thèse de doctorat, Paris, Ecole des Mines de Paris, 1991. 